\title{
EFFECT OF AEROBIC EXERCISES ON QUALITY OF LIFE, RATE OF PERCEIVED EXERTION AND PULMONARY FUNCTION TEST IN HIV FEMALES
}

\author{
Sneha D Ghuman ${ }^{1}$, Shilpa Parab 2 . \\ ${ }^{* 1}$ Assistant professor, MAEER'S Physiotherapy College, Pune, Maharashtra, India. \\ ${ }^{2}$ Principal, CMF's College of Physiotherapy, Pune, Maharashtra, India.
}

\section{ABSTRACT}

Introduction: AIDS (Aquired Immuno Deficiency Syndrome) is caused by a retrovirus called Human Immuno Deficiency Virus (HIV). The profile of HIV infection has changed dramatically since the advent of Highly Active Anti Retroviral Therapy (HAART). These developments have been mirrored by perceived increasing prevalence of impairments, activity limitations and participation restriction for many people living with HIV. There is changing gender trend in HIV cases. Although HAART allows for reduction in HIV related mortality the extension in patient's life can be associated with reduction in Quality of life. Exercise has been proven to health benefits like increased aerobic fitness to improve mood of general population. Aerobic exercise has been reported to reduce negative psychological states, but also to improve an array of positive states.

Materials and Methods: 25 subjects were selected as per the inclusion and exclusion criteria. Written consent was obtained. Subjects were trained at $50-70 \%$ of $\mathrm{HR}_{\max }$ for 3 times a week for 6 week.

Result: Pre and Post data was analyzed using Repeated measures ANOVAs.

Conclusion: Aerobic exercises help to improve Quality of life, Rate of perceived exertion and pulmonary function of females affected by HIV.

KEY WORDS: Acquired Immuno Deficiency Syndrome, Anti Retroviral Therapy, Impairments, Aerobic Fitness.

Address for correspondence: Dr. Sneha Ghuman, Assistant professor, MAEER'S Physiotherapy College, Talegaon Dabhade, Pune, Maharashtra 410507, India. Mobile no.: +919028951449

E-Mail: stunysneha9@gmail.com

\section{Access this Article online}

\section{Quick Response code}

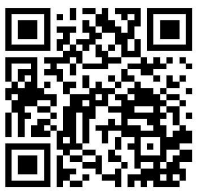

DOI: $10.16965 /$ ijpr.2017.121
International Journal of Physiotherapy and Research

ISSN 2321- 1822

www.ijmhr.org/ijpr.html

Received: 20-02-2017

Accepted: 23-03-2017

Peer Review: 20-02-2017 Published (0): 11-06-2017

Revised: 05-03-2017

Published (P): 11-06-2017

\section{INTRODUCTION}

In India it is estimated that around 2.4 million people are currently living with HIV [1]. The prevalence of HIV at antenatal clinics in Maharashtra was $0.5 \%$ in 2007 [2].

Evaluation of CDC data revealed a changing gender trend in HIV cases, while number of cases in males continue demand concern the percentage of women with HIV is growing rather rapidly in comparison [3]. There are multiple biological, social, economic and environmental risk factors that increase women likely hood of contracting HIV.

Physiology of female genital tract makes it more vulnerable to HIV than men. Social and gender inequalities such as poverty. Unequal educational opportunities force women to rely on male partners for financial support making it more 
difficult for them to insist on intervention that reduce their risk of acquiring HIV.

Considering the discomfort associated with disease progression the social impact of diagnosis the emotional consequences of dealing with the diagnosis of HIV and related stigma and economic hardship faced by many takes it toll on its victims QOL [4-6].

Studies have shown that many women choose not to disclose HIV status for fear of abandonment, rejection, discrimination, violence and accusation of infidelity from their partners, families and communities [6].

With the advent of HAART has dramatically altered the survival rate of persons with HIV/ AIDS in countries where such treatment is available. With the advent of highly active anti-retroviral therapy, new problems have emerged are complicated dosing regimens [7].

These developments due to HAART have been mirrored by perceived increasing prevalence of impairments, activity limitations and participation restriction for many people living with HIV [8]. Some anti-retroviral medications are known to directly increase depressive moods and anxiety in many cases it may be the negative psychological responses instead due to adverse effect of HAART $[9,10]$.

Health related QOL for patients living with HIV is becoming important with the other goals of therapy to reduce the symptoms, suppression of the virus and extension of survival. Adverse effect of potent ART can worsen QOL [11].

Exercise has been proven to health benefits like increased aerobic fitness to improve mood of general population. Aerobic exercise has been reported to reduce negative psychological states, but also to improve an array of positive states. There is growing evidence that exercise is sufficient to increase positive mood state, energy and positive well - being [12].

Exercise has shown to improve strength, cardiovascular function and psychological status in general population, but the effectiveness and safety of aerobic exercise for adults living with HIV infection have not been established. The insufficient number of HIV women subject's makes it difficult to characterize and understand the disease in HIV females and their health status. Hence this study was conducted to find out the effect of aerobic exercises on QOL, Rate of perceived exertion and pulmonary function test in HIV females.

The aim of the study was to study the effect of aerobic exercises on Quality of life, Rate of perceived exertion \& pulmonary function test in HIV females.

Objectives of the study were:(1)To study the effect of aerobic exercises on quality of life using MOS HIV QOL in HIV females.(2)To study the effect of aerobic exercise on rate of perceived exertion in HIV females pre and post aerobic exercises (3)To study the changes in aerobic capacity of HIV females during aerobic training.

The null hypothesis is aerobic exercise does not have effect on Quality of life, rate of perceived exertion and pulmonary function test in HIV females and Alternate hypothesis is aerobic exercise have effect on Quality of life, rate of perceived exertion and pulmonary function test in HIV females

\section{MATERIALS AND METHODS}

The institutional research ethical committee approval was obtained.

Research design: It is Quassy Experimental study.

The patients with HIV stage 1 were included in the study.

The study was conducted at a hospital physiotherapy set-up which receives patients from urban as well as rural areas.

25 subjects were included in the study.

Inclusion Criteria: (1) Patient having HIV stage 1 (2) Age 18-40 yrs (3) female population.

Exclusion Criteria: (1) Having any secondary complications like skin lesions, systemic rashes, and undefined weight loss. (2)Doing exercises regularly. (3) Having any pulmonary, cardiac complications, neurological problems \& musculoskeletal disorders. (4)Pregnant women. (5)Not willing to participate.

Procedure: Subjects were selected as per inclusion and exclusion criteria, 25 could meet the inclusion and exclusion criteria. Nature of the study was explained to them. Written 
consent was obtained. Baseline data was obtained from them in the form of demographic data and information sheet was completed. MOS-HIV QOL questionnaire was completed by interview format. Six minute walk test was conducted and all the three parameters were noted at the start of the test and after 6 min. Rate of perceived exertion were rated on $C^{\prime} R^{\prime} 10$ scale. Subjects were trained at the $50-70 \%$ of HR max on treadmill10 mins warm up followed by 30 mins treadmill and 15 mins cool down exercises for 3 times a week for 6 week. At the end of treadmill session RPE was noted. Aerobic capacity was assessed using a Spirotech clarity spirometer.

Procedure was explained and best of three was considered. All the data was again collected after 3 weeks and at the end of 6 weeks. Subjects were also informed if they have any discomfort they can report during the training. They could stop the training further if they were not comfortable or faced any health issues.

Table 1: Quality of Life (QOL) at baseline, 3 weeks and 6 weeks of training.

\begin{tabular}{|c|c|c|c|c|}
\hline & 0 weeks & 3 weeks & 6 weeks & \\
\hline Mean & 14.58333 & 50 & 77.08333 & P value \\
\hline S.D. & 21.21528 & 0 & 25.44887 & $P<0.005$ \\
\hline
\end{tabular}

S.D standard deviation

$\mathrm{P}<0.005=$ statistically significant

The above table shows statistically significant improvement in Quality of life

Table 2: Rate of perceived exertion at baseline, 3 weeks and 6 weeks.

\begin{tabular}{|c|c|c|c|c|}
\hline & 0 weeks & 3 weeks & 6 weeks & \\
\hline Mean & 7.12 & 5.52 & 4.44 & P value \\
\hline S.D. & 1.201 & 0.9628 & 0.8699 & $P<0.003$ \\
\hline
\end{tabular}

$\mathrm{P}<0.003=$ statistically significant

The above graph shows statistically significant in RPE after 6 weeks

Table 3: Pulmonary function at baseline, 3 weeks and 6 weeks

\begin{tabular}{|c|c|c|c|c|}
\hline & 0 week & 3 week & $\mathbf{6}$ week & \\
\hline Mean & 87.4 & 94.32 & 99.16 & P value \\
\hline S.D. & 5.614 & 3.509 & 2.824 & P $<0.05$ \\
\hline
\end{tabular}

The above table shows $p<0.05$ which is statistically significant.

Statistical Analysis: Mean \& Standard deviation was calculated. All analysis was made using SPSS software version 12. Pre and post data was analyzed using Repeated measures of ANOVAs. All non-parametric data was analyzed using Kruskal Wallis test. Level of significance was set at $p \leq 0.05$

\section{DISCUSSION}

The present study was designed to evaluate the effectiveness of aerobic training program on Quality of life, Rate of perceived exertion, and pulmonary function test in HIV females. The study was conducted on 25 females diagnosed as HIV stage 1.

The results of the study suggests that effect of aerobic exercises on QOL $p<0.005$ which is statistically significant improvement, PFT $p<0.05$ highly significant and on RPE $p<0.003$ which shows statistically significant improvement.

The results indicated that, at least, the exercise program did not cause harm with regards to the immunological function. On the other hand, a significant improvement in perception of life satisfaction was observed for the participants.

There was no previous sample calculation according to the characteristics of the HIVinfected patients. It is known that it is difficult to compose large groups with this type of patient, because of the likelihood of dropouts due to clinical problems.

Moreover, these patients do not always want to have disclosed their condition. For this reason, most of the available studies on the relationship between AIDS and exercise were performed with small samples, such as de Souza et al. (14 patients in the experimental group, without CG), Dolan et al. (40 patients in the experimental group, home-based program, without GC), Driscoll et al. (11 patients in the experimental group and 14 controls), and Roubenoff and Wilson ( 6 patients in the experimental group and 19 controls), MacArthur, Levine and Birk (3 patients in the experimental group and 3 controls), Rigsby et al. (19 patients in the experimental group, without GC) or Spence et al. (24 patients in the experimental group and 12 controls) [13-16].

In general, it is accepted that the preservation of physical and functional fitness contributes to 
a better perception of quality of life.

The improvement of QOL \& aerobic capacity of seropositive individuals observed in this study is consistent with previous researches and confirm their results. The study of LaPerriere et al. may have been the first to examine this relationship. However, the objective of this group was different since the participants were still unaware of their infection. Those who performed aerobic exercises and were seropositive for HIV had lower levels of depression in relation to individuals with the same results but that did not exercise [17].

Aerobic exercise might contribute to the improvement of emotional status, increase the release of endogenous opioids and reduce the activity of the adrenocortical, pituitary and hypothalamic systems (ACPH) [18].

Considering this model, physical activity could moderate the psychological and physiological sequels from chronic diseases, including HIV/ AIDS [19]. It is relevant to mention yet, that the performance of these exercises in group, gathering people with same problems and anxieties, may have been a contribution to the results obtained [20].

Better function is possible, though, by means of exercise as a means to improve aerobic capacity, thus implying better mitochondrial function. The result of our study coincides with the study of Keyser et al 2004 shown that people with HIV to have abnormally lowered capacity to utilize oxygen and perform physical work. It is likely that the etiology of this reduced work capacity is multifactorial, sparse evidence suggests that moderate to high intensity training is effective in improving functional aerobic capacity of HIV positive population [21].

In conclusion, a physical training program of moderate intensity, combining aerobic, strengthening and flexibility exercises was able to significantly improve the perception of life satisfaction of seropositive subjects without causing immunological impairment, at least in the characteristics observed in the present study. Therefore, supervised physical activity programs can be effective tools for improving psychological well-being of patients with HIV.

Thus aerobic exercises help to improve Quality of life, functional status of females affected by HIV.

\section{Conflicts of interest: None}

\section{REFERENCES}

[1]. HIV mortality and infection in India: estimates from nationally representative mortality survey of 1.1 million homes. BMJ 2010;340 doi: 10.1136/ bmj.c621 (Published 23 February 2010)

[2]. National AIDS Control Organization (NACO), National Institute for Health and Family Welfare. Annual HIV sentinel surveillance 2006 India country report. 2007HARSH MOHAN text book of pathology 6 edition

[3]. Cederfjall, C., Langius-Eklof, A., Lidman, K., \& Wredling, R. Genderdifferences in perceived health-related quality of life among patients with HIV infection. AIDS Patient Care and STDs, 2001;15(1):31-39.

[4]. HIV in India - A Complex Epidemic Robert Steinbrook, M.D. N Engl J Med 2007; 356:1089-1093March 15, 2007.

[5]. Kenneth A Cederjfall. Gender differences in health related QOL among patients with HIV infection. AIDS patient care STD's. 2001;15(1)31-39.

[6]. Gender differences in quality of life in persons infected with HIV Bunting, S. Sources of stigma associated with women with HIV. Advances in Nursing Science, 1996;19(2):64-73.

[7]. Ciccolo JT, Jowers EM, Bartholomew JB. The benefits of exercise training for quality of life in HIV/AIDS in the post-HAART era. Sports medicine. $2004 \mathrm{Jul}$ 1;34(8):487-99.

[8]. O'Brien K, Nixon S, Tynan AM, Glazier RH. Aerobic exercise interventions for people living with HIV/ AIDS: Implications for practice, education, and research. Physiotherapy Canada. 2006 Apr;58(2):11429. DOI 10.2310/6640.2006.00032

[9]. HIV Classification: CDC and WHO Staging Systems Guide for HIV/AIDS Clinical Care, HRSA HIV/AIDS WHO/HIV/2005.02

[10]. Feigenbaum K, longstaff $L$. Management of the metabolic syndrome in patients with HIV. The diabetes educator.2010;36(3)457-464.

[11]. Bopp, C., Phillips, K., Fulk, L., \& Hand, G. Clinical implications of therapeutic exercise in HIV/AIDS. Journal of the Association of Nurses in AIDS Care. 2003;14(1):73-78.

[12]. Maman. S. Medley AM, Gurcia Barriers And outcome of HIV serostatus disclosure among women in developing countries. Implication for prevention of mother to child transmission program. 2004;82(4):299-307.

[13]. Gregory A Hand, Kenneth D Phillips moderate intensity exercise training reverses functional aerobic impairments in HIV infected individuals. AIDS Care 2008;20(9):1066-1074.

[14]. Deresz LF , Spirinz E, Kramer AS et al, regulation of oxidative stress in response to acute aerobic and resistance exercise $n$ HIV infected subjects: a case control study. AIDS Care 2010;22:1410-1417. 
[15]. Chapplain JM, Beilot J, Begue JM et al. Mitochondrial abnormalities in HIV infected lipoatropic patients treated with anti-retroviral agents. J Acquir Immune deficiency Syndrome 2004;37:1477-1488.

[16]. Dolan SE, Fronera W, Librizzi J, Ljungquist K, Juan S, Dorman R, et al. Effects of a supervised home-based aerobic and progressive resistance training regimen in women infected with human immunodeficiency virus: a randomized trial. Arch Intern Med. 2006;166(11):1225-31.

[17]. Miller TL, Somarriba G, Kinnamon DD et al, The effect of a structured exercise program on nutrition and fitness outcome in human-immunodeficiency virus-infected children. AIDS Research and Human Retroviruses. 2010;26(3):313-319.

[18]. Cade W T, Fautry L.E Nabar S R a comparision of QT And a VO2 in individual with HIV taking and not taking HAART. Med And science in sports and exercise. 2003;35(7):1108-1117.
[19]. Hicks C Currier J Sax P Current challenges in HIV Tolerability of anti-retroviral and metabolic complications. AIDS ppatient care STD'S 2003;17:221233.

[20]. Joseph T Ciccolo, esbelle M. Jowers : The benefits of Exercise training for Quality of life in HIV/AIDS iin the post HAART era. Sports med 2004;34(8):487-499.

[21]. Hand GA impact of aerobic and resistance exercise on health of HIV infected persons AMJ lifestyle MED 2001;3:489-499.

How to cite this article:

Sneha D Ghuman, Shilpa Parab. EFFECT OF AEROBIC EXERCISES ON QUALITY OF LIFE, RATE OF PERCEIVED EXERTION AND PULMONARY FUNCTION TEST IN HIV FEMALES. Int J Physiother Res 2017;5(3):2061-2065.

DOI: $10.16965 /$ ijpr.2017.121 